\title{
Reduced density matrix approach to phononic dissipation in friction
}

\author{
A. Özpineci, ${ }^{1}$ D. M. Leitner, ${ }^{2}$ and S. Ciraci ${ }^{1}$ \\ ${ }^{1}$ Department of Physics, Bilkent University 06533 Ankara, Turkey \\ ${ }^{2}$ Department of Chemistry, University of Nevada, Reno, Nevada 89557
}

(Received 9 September 1999)

\begin{abstract}
Understanding mechanisms for energy dissipation from nanoparticles in contact with large samples is a central problem in describing friction microscopically. Calculation of the reduced density matrix appears to be the most suitable method to study such systems that are coupled to a large environment. In this paper, the time evolution of the reduced density matrix has been evaluated for an arbitrary system coupled to a heat reservoir. The formalism is then applied to study the vibrational relaxation following the stick-slip motion of an asperity on a surface. The frequency and temperature dependence of the relaxation time is also determined. Predictions of the reduced density matrix are compared with those obtained by using the Golden Rule approach.
\end{abstract}

\section{INTRODUCTION}

Friction and its microscopic origins have been intensively investigated in recent years. ${ }^{1}$ Progress in atomic force microscopy (AFM) has made possible precise force measurements at the atomic scale. ${ }^{2}$ These data, together with simulations, theory and ab initio force calculations have helped to provide a detailed picture of friction. ${ }^{3-13}$ Much of this work is concerned with the nature and rate of energy transfer from lubricant layers or asperities into bulk substrates. The understanding of such processes has implications as far-ranging as the design of solar collectors, ${ }^{14}$ where collected energy must be transferred to the bulk before re-emission to the surroundings; to the understanding of reactions in living cells catalyzed on small surfaces, ${ }^{15}$ as well as biomolecular motors; ${ }^{16}$ and to the design of lubricants for industrial purposes. ${ }^{16}$ In many cases, friction is the result of energy dissipation by multiphonon processes. We address in this article energy dissipation in a stick-slip model for friction involving small and sparsely distributed asperities on a surface. Previous atomic scale studies of friction assumed that the mechanical energy stored in the stick stage dissipates totally within the slip stage. ${ }^{17}$ Since the distance between the stick and slip stages can be as small as a lattice parameter of substrate surface, how fast the stored mechanical energy dissipates is a central problem in achieving a microscopic understanding of friction. In this work we determine the nature and calculate the rate of damping of the adsorbed molecule, nanoparticle or asperity following the slip step.

Relaxation processes of adsorbates have been studied both theoretically and experimentally (see, e.g., Refs. 1824). In most of these works, the authors used either phenomenological approximations ${ }^{20}$ or assumed that the system under study is at least at quasiequilibrium and used equilibrium theory to study their properties. ${ }^{18,19,22,23}$ In this article, a Redfield theory-like approach is developed (for the derivation of the Redfield theory and some of its applications see, e.g., Refs. 25-27) to calculate the time evolution of the reduced density matrix of an adsorbate on a substrate within the framework of a stick-slip model for friction.

Consider an object adsorbed on the surface of a sample, a metal or insulator, with vibrational frequency $\Omega$. In general there are two possible decay modes: 1) it can create elec- tronic excitations in a metal, e.g., create electron-hole pairs, or 2) it can create phononic excitations. In this article our interest will be in phononic dissipation. If $\Omega \simeq n \omega_{0}$ where $\omega_{0}$ is the maximum phonon frequency of the sample, the excitations can decay only by the creation of $n$ phonons in the sample. ${ }^{20}$ For large $n$, this contribution is in general negligible. Thus for small particles on a solid substrate, for which the vibrational frequency of the adsorbate might be much higher than the band of substrate mode frequencies, energy dissipation from the adsorbate into the substrate may be quite slow. For such cases, anharmonic coupling between the adsorbate and substrate gives rise to energy flow into the substrate. For example, for $\mathrm{CO}$ adsorbed on $\mathrm{Cu}$, the $\mathrm{Cu}-\mathrm{CO}$ stretch vibration is $\Omega \simeq 1.5 \omega_{0}$ and decay by creating two phonons might be an important mechanism for the vibrational damping of the molecule. We will address the problem of phononic energy dissipation in friction following stickslip motion of an adsorbate whose vibrational frequency lies above the band of substrate frequencies, like $\mathrm{CO}$ on a $\mathrm{Cu}$ surface. In Ref. 23 two and three phonon contributions to the dissipation of the $\mathrm{Cu}-\mathrm{CO}$ stretch vibrations was studied using the Golden Rule formula. We will study this and similar systems here using a more general Redfield theory-like approach for various $\Omega$ 's to understand the dependence of the dissipation rate on the coupling between the sample and the adsorbed atom. We assume that the adsorbates are sparsely distributed so that we can neglect interactions among them.

The organization of the paper is as follows: In Sec. II, we calculate the time evolution of the reduced density matrix which allows one to take into account all nonequilibrium properties of a system and also takes into account possible coherence and incoherence effects (for the properties of density matrices see, e.g., Ref. 27). Possible limitations on the obtained evolution are also discussed. In Sec. III a model system is proposed which is relevant for the energy dissipation in the stick-slip motion of atomic scale friction. This model is analyzed and the energy dissipation therefrom is calculated by using the reduced density matrix theory in Sec. IV. Concluding remarks are given in Sec. V.

\section{EVOLUTION OF THE REDUCED DENSITY MATRIX}

In studying the dynamics of systems coupled to an environment, it is most natural to use the reduced density matrix 
(RDM) formalism. The RDM, $\rho_{R}$, is obtained from the density matrix, $\rho$, of the system plus the environment by taking its trace over the environmental degrees of freedom,

$$
\rho_{R}(t)=\operatorname{Tr}_{b} \rho(t),
$$

where $\operatorname{Tr}_{b}$ denotes trace over the degrees of freedom of the environment. In the following, the index $R$ is omitted whenever there is no possibility of confusion. Once the time evolution of the RDM of the system is known, the time evolution of the expectation value of any observable, $\mathcal{O}$, of the system can be obtained as:

$$
\langle\mathcal{O}\rangle(t)=\operatorname{Tr}\left[\rho_{R}(t) \mathcal{O}\right]
$$

The time dependence of the RDM of the system can be obtained from the definition of the RDM in Eq. (1). Let

$$
\mathcal{H}=\mathcal{H}_{s}+\mathcal{H}_{r}+\mathcal{H}_{\text {int }},
$$

where $\mathcal{H}_{s}, \mathcal{H}_{r}$ are the system and reservoir Hamiltonians, respectively, and $\mathcal{H}_{\text {int }}$ describes the interaction between them. We will assume, without loss of generality, that

$$
\mathcal{H}_{\text {int }}=\sum_{s} Q_{s} F_{s}
$$

where $Q_{s}\left(F_{s}\right)$ acts only on the system (reservoir) degrees of freedom. The time evolution of the components of the RDM is given by

$$
\rho_{\alpha \beta}(t)=\rho_{\alpha \beta}(0) e^{-i \omega_{\alpha \beta} t}+\sum_{\alpha^{\prime} \beta^{\prime}} R_{\alpha \alpha^{\prime} ; \beta \beta^{\prime}}(t) \rho_{\alpha^{\prime} \beta^{\prime}}(0) e^{-i \omega_{\alpha \beta^{\prime}} t},
$$

where $\hbar \omega_{\alpha \beta}=\epsilon_{\alpha}-\epsilon_{\beta}$ and the tensor $R_{\alpha \alpha^{\prime} ; \beta \beta^{\prime}}(t)$ is defined as

$$
\begin{aligned}
R_{\alpha \alpha^{\prime} ; \beta \beta^{\prime}}(t)= & \sum_{i j} P\left(E_{j}\right)\left\langle\alpha i|\mathcal{S}(t)| \alpha^{\prime} j\right\rangle\left\langle\beta^{\prime} j\left|\mathcal{S}^{\dagger}(t)\right| \beta i\right\rangle \\
& -\delta_{\alpha \alpha^{\prime}} \delta_{\beta \beta^{\prime}},
\end{aligned}
$$

where the scattering matrix, $\mathcal{S}(t)$, is defined as

$$
\begin{aligned}
\mathcal{S}(t)= & e^{i / \hbar \mathcal{H}_{0} t} e^{-i / \hbar \mathcal{H} t}=1-\frac{i}{\hbar} \int_{0}^{t} d t^{\prime} \mathcal{H}_{\text {int }}\left(t^{\prime}\right) \\
& +\left(-\frac{i}{\hbar}\right)^{2} \int_{0}^{t} d t_{1} \int_{0}^{t_{1}} d t_{2} \mathcal{H}_{\text {int }}\left(t_{1}\right) \mathcal{H}_{\text {int }}\left(t_{2}\right)+\ldots
\end{aligned}
$$

Here, $\mathcal{H}_{0}$ is defined to be $\mathcal{H}_{0}=\mathcal{H}_{r}+\mathcal{H}_{s}$. Also $\mathcal{H}_{\text {int }}(t)$ $=e^{i / \hbar \mathcal{H}_{0} t} \mathcal{H}_{\text {int }} e^{-i / \hbar \mathcal{H}_{0} t}$, and $|\gamma j\rangle=|\gamma\rangle \otimes|j\rangle$ with,

$$
\begin{gathered}
\mathcal{H}_{s}|\gamma\rangle=\epsilon_{\gamma}|\gamma\rangle, \\
\mathcal{H}_{r}|j\rangle=E_{j}|j\rangle .
\end{gathered}
$$

In the following Greek (Latin) letters will denote the system (reservoir) degrees of freedom. In deriving this result it is assumed that the reservoir is always in equilibrium so that the density matrix of the whole system can be factorized as

$$
\langle\gamma j|\rho(t)| \delta k\rangle=\delta_{j k} \rho_{R \gamma \delta} P\left(E_{j}\right),
$$

where the diagonal density matrix elements of the reservoir are defined as

$$
P\left(E_{j}\right)=\frac{e^{-\beta E_{j}}}{Z},
$$

where $Z=\Sigma_{j} e^{-\beta E_{j}}$.

Until this point, the only assumption made is that the density matrix of the whole system is factorizable which resulted in a linear equation for the components of the RDM. The applicability of this approximation should be studied carefully. This assumption is valid only if there exists a weak coupling between the system and the reservoir so that the tensor product states $|\alpha j\rangle$ can be considered to be nearly the eigenstates of the whole system. If there is strong coupling between the system and the reservoir, or if the "reservoir' is a finite one, the density matrix of the whole system in general cannot be factorized and one has to do without this simplifying approximation. This approximation should be valid for our study of phononic energy decay from an adsorbate coupled anharmonically to a large substrate.

Now, the main task is to find a suitable approximation for the tensor $R_{\alpha \alpha^{\prime} ; \beta \beta^{\prime}}(t)$. Once it is known, the time evolution of the RDM can be calculated. Unfortunately, the expression obtained by straightforward application of the second order expansion of the $\mathcal{S}$ matrix yields a result which is valid only if the time, $t$, is sufficiently short. To overcome this difficulty we used an iterative scheme in which we calculated the initial RDM and then evolved it one step in time; taking the evolved RDM as the initial RDM, we evolved it one step further. At each step, we evolved for a short enough time. Since energy is not conserved for finite times, one has to impose energy conservation by hand. For this reason, the matrix elements of $\mathcal{H}_{\text {int }}$ coupling states of different energies are neglected. The calculations are similar to the ones done in scattering theory with the result

$$
\begin{aligned}
\rho_{\alpha \beta}(t+\Delta t)= & \rho_{\alpha \beta}(t) e^{-i \omega_{\alpha \beta} \Delta t} \\
& +\sum_{\alpha^{\prime} \beta^{\prime}} R_{\alpha \alpha^{\prime} ; \beta \beta^{\prime}}(\Delta t) \rho_{\alpha^{\prime} \beta^{\prime}}(t) e^{-i \omega_{\alpha \beta} \Delta t},
\end{aligned}
$$

$$
\begin{aligned}
R_{\alpha \alpha^{\prime} ; \beta \beta^{\prime}}(\Delta t)= & \frac{\Delta t}{\hbar^{2}} \delta_{\alpha-\alpha^{\prime}, \beta-\beta^{\prime}}\left(\sum_{s s^{\prime}} Q_{s}^{\alpha \alpha^{\prime}} Q_{s^{\prime}}^{\beta^{\prime} \beta} j_{s s^{\prime}}\left(\omega_{\alpha \alpha^{\prime}}\right)\right. \\
& -\frac{1}{2} \delta_{\alpha \alpha^{\prime}} \sum_{s s^{\prime}} \sum_{\gamma} Q_{s}^{\beta \gamma} Q_{s^{\prime}}^{\gamma \beta} j_{s s^{\prime}}\left(\omega_{\gamma \beta}\right) \\
& \left.-\frac{1}{2} \delta_{\alpha \alpha^{\prime}} \sum_{s s^{\prime}} \sum_{\gamma} Q_{s}^{\alpha \gamma} Q_{s^{\prime}}^{\gamma \alpha} j_{s s^{\prime}}\left(\omega_{\gamma \alpha}\right)\right)
\end{aligned}
$$

$$
j_{s s^{\prime}}(\omega)=\Delta t \sum_{k j}^{\prime} P\left(E_{j}\right) F_{s}^{k j} F_{s^{\prime}}^{j k},
$$

where the prime on the summation in Eq. (13) indicates that the sum should be carried out over states for which $\hbar \omega$ $=E_{j k}$. 


\section{THE MODEL HAMILTONIAN}

Consider an object adsorbed on a surface. Let $M$ be the mass of a reservoir atom and $m$ be the mass of the adsorbed object. Assume that the small adsorbate is bonded to a single atom of the sample and the interaction between the sample atom and the adsorbed atom is described by the Morse potential,

$$
U(u-v)=E_{0}\left\{e^{-2 \alpha(u-v)}-2 e^{-\alpha(u-v)}\right\},
$$

where $u$ and $v$ are the vertical displacements of the adsorbed atom and the sample atom, respectively. $E_{0}$ is the binding energy of the adsorbed atom and $\alpha$ can be related to the vibrational frequency, $\Omega$, of the adsorbed atom through

$$
\alpha=\left(\frac{m \Omega^{2}}{2 E_{0}}\right)^{1 / 2} .
$$

Expanding the potential and retaining the lowest order terms, we get

$$
\mathcal{H}_{\text {int }}=A u v+B u v^{2},
$$

where

$$
\begin{aligned}
& A=-2 E_{0} \alpha^{2}, \\
& B=-3 E_{0} \alpha^{3} .
\end{aligned}
$$

Note that the $u^{2} v$ term obtained from the expansion of the potential is not included. Such a term gives rise to the absorption (emission) of one bulk phonon and emission (absorption) of two localized, atomic phonons. Since we are mostly concerned with $\Omega>\omega_{0}$, i.e., adsorbate frequencies higher than bulk frequencies, as discussed below, we neglect this term. We note that for $\Omega>\omega_{0}$, the $u v$ term makes no contribution to dissipation since it does not conserve energy, and we only have the $u v^{2}$ term. For the other case, $\Omega$ $<\omega_{0}$, the contribution to the decay rate from the $u v$ term is generally much larger than from the $u v^{2}$ term. The decay of the vibrational excitation of the adsorbate to the substrate for the case where $\Omega<\omega_{0}$ using only harmonic coupling has been studied by exact diagonalization of the Hamiltonian. ${ }^{28}$ The calculated value for the decay rate in Ref. 28 is not surprisingly greater than the value we have calculated in Sec. IV (by two orders of magnitude), where we used only the $u v^{2}$ term. In this article, we consider only effects of the $u v^{2}$ term. We thus focus on energy dissipation for highfrequency adsorbates, where $\Omega>\omega_{0}$. Then the full phononic Hamiltonian of the system we study becomes

$$
\mathcal{H}=\hbar \Omega b^{\dagger} b+\sum_{k \sigma} \hbar \omega_{k \sigma} b_{k \sigma}^{\dagger} b_{k \sigma}+B u v^{2}
$$

where $\omega_{k \sigma}$ are the frequencies of the sample phonons with wave vector $k$ and polarization vector $e_{\sigma}$, and $b$ and $b_{k \sigma}$ are the annihilation operators for the phonons at the adsorbed atom and the phonons in the sample, respectively.

\section{NUMERICAL ANALYSIS AND DISCUSSION}

In this section, the evolution of the density matrix will be analyzed numerically. In order to construct the initial density matrix, consider the following situation: Assume that two
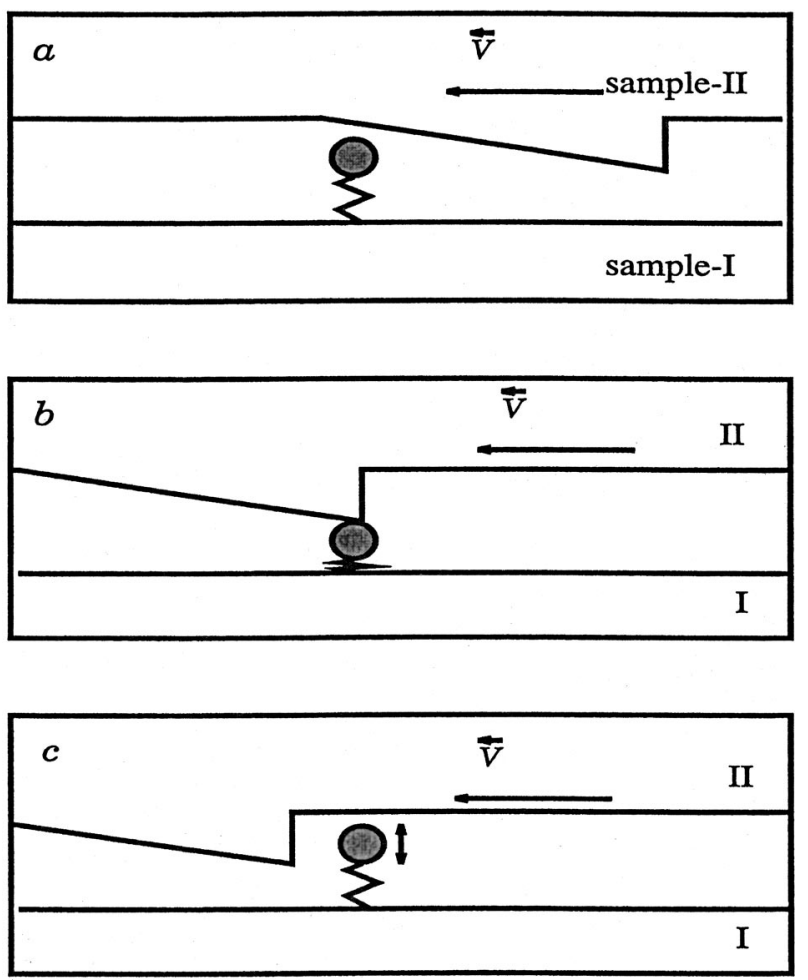

FIG. 1. An asperity between the surfaces of two samples, one of which moves with a velocity v. (a) There is no interaction between Sample II and the rest of the system. (b) The asperity is squeezed by corrugations on the surface of Sample II, absorbing some of the translational energy of Sample II. (c) The asperity is suddenly released causing it to oscillate about a fixed location on Sample I; the interaction between Sample II and the rest of the system can again be neglected. We focus on the damping of the vibrational energy of the asperity by Sample I.

samples, Sample I and Sample II, are moving on top of one another with an adsorbed layer on the bottom one, and there is no direct interaction between the samples, as illustrated in Fig. 1. Consider the case when the coverage of the adsorbed layer is so low that the interactions between the adsorbates can be neglected, in which case one can treat each adsorbate independently. Each adsorbate, or asperity is strongly bound to Sample I and hence moves vibrationally about a fixed location on Sample I. During the motion of Sample II, the adsorbate or asperity on Sample I will be pushed and released. The atom will be adiabatically pushed down, due to the wedge shape or corrugation of the surface, displacing it from its equilibrium position and storing energy in it. Then it is suddenly released. After its release there is no interaction between the asperity and Sample II. This stick-slip model is relevant for energy dissipation through phonons in dry sliding friction or lubrication, as well as for the vibrations of the adsorbed species. The character of the contribution of such a mechanism to friction between the bodies would depend on the rate of relaxation of this nonequilibrium situation.

Initially, the density matrix of the system plus reservoir is the equilibrium density matrix

$$
\rho=\sum_{\alpha j} \frac{e^{-\beta\left(\epsilon_{\alpha}+E_{j}\right)}}{Z}|\alpha j\rangle\langle\alpha j|,
$$


where $Z=\Sigma_{\alpha j} e^{-\beta\left(\epsilon_{\alpha}+E_{j}\right)}, \alpha$ denotes the number of phonons of the asperity and $j$ is a multiple index describing the number of phonons in each mode, $\mathbf{k} \sigma$, of the sample.

Adiabatically displacing the asperity would not cause it to go out of equilibrium. The density matrix will still be diagonal with the same diagonal elements but in the displaced basis

$$
\rho=\sum_{\alpha j} \frac{e^{-\beta\left(\epsilon_{\alpha}+E_{j}\right)}}{Z}\left|\alpha^{\prime} j\right\rangle\left\langle\alpha^{\prime} j\right|
$$

with the same $Z$. The displaced harmonic oscillator states, $\left|\alpha^{\prime}\right\rangle$, are defined as

$$
\left|\alpha^{\prime}\right\rangle=e^{i / \hbar s \hat{p}}|\alpha\rangle=\sum_{\beta} c_{\alpha^{\prime} \beta}|\beta\rangle,
$$

where $s$ is the displacement of the oscillator and $\hat{p}$ is the momentum operator of the adsorbate. When the asperity is suddenly released, the density matrix does not change, but now, in the absence of the external force due to Sample II, the density matrix is no longer diagonal in the energy eigenstates, and the adsorbate is out of equilibrium. Denoting the RDM of the system right after it is released by $\rho\left(0^{+}\right)$, we have

$$
\rho\left(0^{+}\right)=\operatorname{Tr}_{b} \sum_{\alpha j} \frac{e^{-\beta\left(\epsilon_{\alpha}+E_{j}\right)}}{Z}\left|\alpha^{\prime} j\right\rangle\left\langle\alpha^{\prime} j\left|=\sum_{\beta \gamma} \rho_{\beta \gamma}(0)\right| \beta\right\rangle\langle\gamma|,
$$

where $\rho_{\beta \gamma}(0)=Z^{-1} \sum_{\alpha} c_{\alpha^{\prime} \beta} c_{\alpha^{\prime} \gamma}^{*} e^{-\beta \epsilon_{\alpha}}$.

Following Ref. 26, we take

$$
\begin{gathered}
u=\left(\frac{\hbar}{2 m \Omega}\right)^{1 / 2}\left(b+b^{\dagger}\right), \\
v=\sum_{k \sigma}\left(\frac{\hbar}{2 m \omega_{k \sigma} N}\right)^{1 / 2}\left(b_{k \sigma}+b_{k \sigma}^{\dagger}\right) \hat{z} \cdot e_{k \sigma},
\end{gathered}
$$

where $N$ is the number of the Sample I atoms and $e_{k \sigma}$ is the polarization vector of mode $k \sigma$. As is pointed out in Ref. 23, this expression for $v$ does not account for the surface which might reflect bulk phonons, and also does not take into account any surface phonons. In order to evaluate the time evolution of the RDM, first the function $j_{s s^{\prime}}(w)$, defined in Eq. (13), has to be evaluated. Note that in our case, $s$ and $s^{\prime}$ can take just a single value, $s=s^{\prime}=1$. Using the expansions given in Eqs. (24) and (25), and choosing

$$
F_{1}=v^{2}
$$

$$
Q_{1}=B u,
$$

we obtain

$$
\begin{aligned}
j_{11}(\omega)=j(\omega)= & \theta(\omega)\left[\left(\frac{\hbar}{2 M}\right)^{2} \int d \omega^{\prime} g\left(\omega^{\prime}\right) g\left(\omega^{\prime}-\omega\right) 4 \frac{\left(n_{B}\left(\omega^{\prime}\right)+1\right) n_{B}\left(\omega^{\prime}-\omega\right)}{\omega^{\prime}\left(\omega^{\prime}-\omega\right)}\right. \\
& \left.+\left(\frac{\hbar}{2 M}\right)^{2} \int d \omega^{\prime} g\left(\omega^{\prime}\right) g\left(\omega-\omega^{\prime}\right) \frac{n_{B}\left(\omega^{\prime}\right) n_{B}\left(\omega^{\prime}-\omega^{\prime}\right)}{\omega^{\prime}\left(\omega-\omega^{\prime}\right)}\right] \\
& +\theta(-\omega)\left[\left(\frac{\hbar}{2 M}\right)^{2} \int d \omega^{\prime} g\left(\omega^{\prime}\right) g\left(\omega^{\prime}-\omega\right) 4 \frac{\left(n_{B}\left(\omega^{\prime}\right)+1\right) n_{B}\left(\omega^{\prime}-\omega\right)}{\omega^{\prime}\left(\omega^{\prime}-\omega\right)}\right. \\
& \left.+\left(\frac{\hbar}{2 M}\right)^{2} \int d \omega^{\prime} g\left(\omega^{\prime}\right) g\left(\omega-\omega^{\prime}\right) \frac{n_{B}\left(\omega^{\prime}\right) n_{B}\left(\omega-\omega^{\prime}\right)}{\omega^{\prime}\left(\omega-\omega^{\prime}\right)}\right],
\end{aligned}
$$

where the integration region in each integration is where the density of states is nonzero and $\omega^{\prime}$ is positive. In this result we have assumed the thermodynamic limit and neglected $\mathcal{O}(1 / N)$ terms. In this study, $g(\omega)$ is represented by the Debye density of states

$$
g(\omega)=\frac{3 \omega^{2}}{\omega_{D}^{3}} \theta\left(1-\frac{\omega}{\omega_{D}}\right)
$$

where $\omega_{D}$ is the Debye frequency, which is $\omega_{0}$, the maximum frequency of the substrate. In order to obtain Eq. (28) from Eq. (13), the summations over states are converted to integrations over energies and the integration region is chosen so that only a small energy violation, $\Delta \omega$, is allowed, which is assumed to satisfy $\Delta \omega \Delta t=1$ from the energy-time uncertainty relation. If one compares Eq. (28) with similar results found in the literature (e.g., Ref. 25), there is an extra factor of $\pi$ which arises because of the assumption that $\Delta t$ is large enough so that one can take the limit $t \rightarrow \infty$ in certain integrals. This factor is not related to the formalism but is just related to the evaluation of Eq. (13). Here, one obtains an iterative equation for the evaluation of the RDM by substituting Eqs. (28) and (12) into Eq. (11). Given an initial density matrix at $t=t_{0}$, one can calculate the density matrix at the time $t_{0}+\Delta t$ using Eq. (11).

The final result can be compared with the results in Ref. 23. In Ref. 23 it is assumed from the beginning that only the diagonal element of the density matrix corresponding to the first excited state, $\rho_{11}$, is nonzero. In this case, the contribution of the other elements of the density matrix can be neglected in the evolution of $\rho_{11}$, and we obtain 


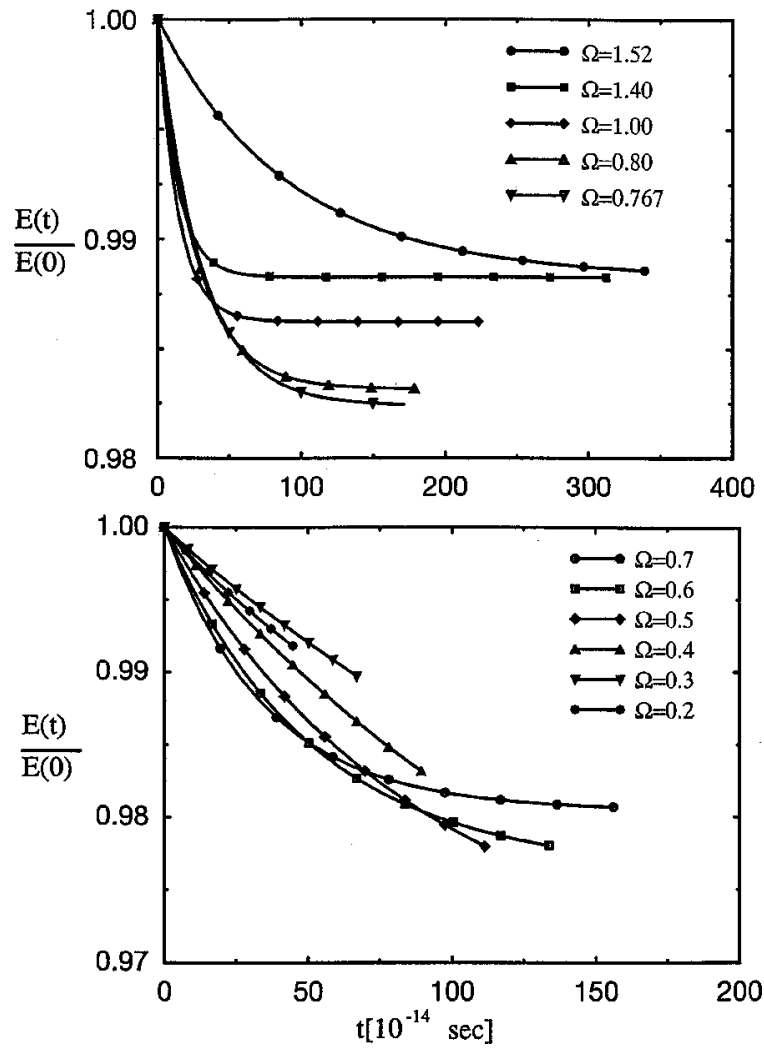

FIG. 2. Calculated decay profiles, $E(t) / E(0)$, for the energy of the vibrating atom for various $\Omega$ 's.

$$
\rho_{11}(t+\Delta t)=\rho_{11}(t)+R_{11 ; 11}(\Delta t) \rho_{11},
$$

which yields a decay rate $-R_{11 ; 11} / \Delta t$, which is nothing but the result derived in Ref. 23 using the Golden Rule (other than an overall factor of $\pi$, as discussed earlier). This feature is quite general in the sense that as long as just the first few elements of the density matrix are important, and for sufficiently low temperatures, the results obtained using this formalism and those obtained by the Golden Rule are almost identical.

We turn now to the numerical calculation of energy dissipation from the asperity into the bulk following the slip step. For the numerical data, we have used the following values: $\hbar \omega_{D}=37.6 \mathrm{meV} ; \quad M=28 \mathrm{amu} ; \quad m=28 \mathrm{amu} ; \hbar \Omega_{0}$ $=46 \mathrm{meV} ; E_{0}=1.8 \mathrm{eV} ; F=10^{-10} \mathrm{~N}$ and $T=300 \mathrm{~K}$. Here $F$ is the maximum vertical force applied to the adsorbed atom and is related to the vertical displacement, $s$, through

$$
s=\frac{F}{m \Omega^{2}} .
$$

We vary the adsorbate frequency $\Omega$ from $0.2 \Omega_{0}$ up to $1.52 \Omega_{0}$, and take the iteration step to be $\Delta t=\Omega^{-1}$. We introduce these parameters into Eq. (11), which we then solve at each time step, $\Delta t$.

In Fig. 2, we have plotted the decay profiles, $E(t) / E(0)$, i.e., the time-dependent energy in the adsorbate mode over its initial energy, for various values of the adsorbate frequency, $\Omega$. In each case the range of the time axis corresponds to 300 iterations, each iteration corresponding to a time of $\Omega^{-1}$. The exponential character of the decay is clear.

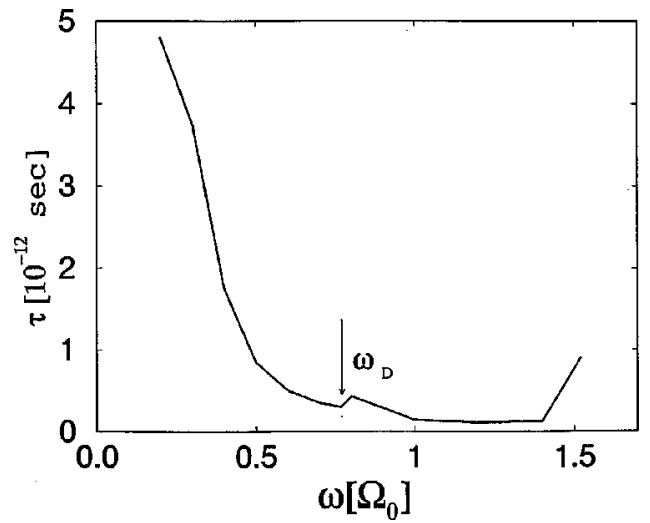

FIG. 3. Dependence of the relaxation time, $\tau$, on the vibrational frequency, $\Omega$, of the adsorbate.

For the numerical calculations, we used only a finite, 16 $\times 16$, part of the infinite density matrix. This caused the matrix elements at the edges to evolve incorrectly. However, as long as they are negligible compared to the matrix elements corresponding to the first few excited states, this does not affect the general profile of the time dependence of the energy, which is mainly determined by the evolution of the first few diagonal elements of the density matrix. In most cases after 300 iterations the matrix elements at the edge become non-negligible. In all cases, we found that the excess energy can be fit almost perfectly to the expression

$$
\Delta E(t)=\Delta E(0) e^{-t / \tau},
$$

where $\Delta E(t)=E(t)-E(\infty)$. Here, $\tau$ is the decay time constant (or the relaxation time).

In Fig. 3, the dependence of the decay time, $\tau$, on the adsorbate frequency, $\Omega$, is shown. In the graph, the frequencies are given in units of $\Omega_{0}$, the representative value of the $\mathrm{CO}-\mathrm{Cu}$ frequency given above. We note that our calculations only include cubic anharmonic coupling of the adsorbate to the substrate. This is reasonable when $\Omega>\omega_{D}$. When the adsorbate frequency is smaller than the Debye frequency, indicated in the figure, the plotted $\tau$ will be too large, since we have neglected linear coupling terms, which are important when $\Omega<\omega_{D}$. We nevertheless include the regime where $\Omega<\omega_{D}$ to show how the cubic anharmonic contribution to $\tau$ varies in this regime, though the focus of this study is where the adsorbate frequency lies outside the band of substrate frequencies.

We see that both for large $\Omega$ and small $\Omega, \tau$ diverges. In the large $\Omega$ limit, the reason is due to the phase space factors; the two phonons created or adsorbed have to be in a band of width $2 \omega_{D}-\Omega$ which goes to zero as $\Omega \rightarrow 2 \omega_{D}$. For $\Omega$ $>2 \omega_{D}$, the adsorbed atom cannot decay through the emission of two phonons and one has to consider three or more phonon processes. In the small $\Omega$ limit, the coupling constant $B$ becomes very small and the system behaves almost as if it is isolated, and cannot decay. In Fig. 3, one also sees that in the region $\Omega \simeq \omega_{D}$ there is an interesting variation in $\tau$. For $\Omega<\omega_{D}$ there is a contribution to the decay process whereby 
TABLE I. Relaxations times, $\tau\left(T, \Omega=\Omega_{0}\right)$, calculated for various temperatures by using RDM and the Golden Rule approach when the frequency of the adsorbate is fixed at $\Omega=\Omega_{0}$.

\begin{tabular}{ccc}
\hline \hline$T(\mathrm{~K})$ & $\mathrm{RDM}\left(10^{-13} \mathrm{sec}\right)$ & Golden Rule $\left(10^{-13} \mathrm{sec}\right)$ \\
\hline 100 & 4.48 & 1.10 \\
200 & 3.63 & 0.44 \\
300 & 1.40 & 0.28 \\
600 & 1.20 & 0.11 \\
900 & 1.12 & 0.05 \\
\hline \hline
\end{tabular}

the adsorbate absorbs a low energy phonon from the sample which unites with an adsorbate phonon to emit a high energy phonon into the sample. This process is absent when $\Omega$ $>\omega_{D}$, which causes $\tau$ to rise when $\Omega$ exceeds $\omega_{D}$. We note that this rise, followed by a drop in $\tau$ with larger $\Omega$, is not affected by the neglect of linear coupling terms since this interesting nonmonotonic variation of $\tau$ with $\Omega$ occurs where the adsorbate frequency is higher than the band of substrate frequencies.

Having discussed the frequency dependence of the relaxation time, we consider another important issue, i.e., the temperature dependence of the dissipation rate. Two questions which have a close bearing on the friction are how the relaxation time varies with the local temperature and to what extent the temperature dependence is taken into account by the Golden Rule approach. Since the latter is only an approximation to the RDM, it is useful to compare the temperature dependence of the relaxation time predicted by RDM with that obtained by the Golden Rule approach, as in Ref. 23. This way we also expect to reveal the limitation of the Golden Rule approach. To this end, we calculate $\tau\left(T, \Omega=\Omega_{0}\right)$ by using two different approaches, i.e., RDM and Golden Rule expression given in Ref. 23. Results of our calculations are listed in Table I. It is seen that $\tau_{\mathrm{GR}}(T=900) / \tau_{\mathrm{GR}}(T=100)=0.05$ and $\tau_{\mathrm{RDM}}(T=900) / \tau_{\mathrm{RDM}}(T=100)=0.25$ and also $\tau_{\mathrm{RDM}}(T$ $=900) / \tau_{\mathrm{GR}}(T=900)=22.4$. The conclusion that one can derive from the results is that the temperature dependence of the relaxation time is underestimated and the dissipation rate is overestimated at high temperatures by the Golden Rule approach.

\section{CONCLUDING REMARKS}

In this work, we developed a formalism based on the reduced density matrix to study the dissipation of excess energy of a vibrating adsorbate into a substrate following compression by another surface, as depicted in Fig. 1. Our focus was on adsorbate frequencies that lie outside the band of substrate frequencies. In this case, anharmonic coupling between the adsorbate and the substrate controls the rate of energy dissipation, and we introduced cubic terms explicitly in our calculations. This work presents an extension and generalization of earlier work in which a Golden Rule formula was used to calculate the rate of energy dissipation from an adsorbate following the slip step. ${ }^{13}$ In that work, the adsorbate was linearly coupled to the substrate, and anharmonic terms were not considered. In this article, we found that at room temperature excess energy decays essentially exponen- tially from the adsorbate into the substrate, using the reduced density matrix formalism with anharmonic coupling and a range of parameters that includes those for $\mathrm{CO}$ adsorbed on a $\mathrm{Cu}$ substrate at room temperature. We have also pointed out the limits of the Golden Rule approach used for a similar system. ${ }^{23}$ At temperatures above $100 \mathrm{~K}$ additional elements of the density matrix contribute to the dissipation rate, contributions not embodied in the Golden Rule approach. We found that the Golden Rule approach overestimates the dissipation rate at high temperatures. Our model is basic and incorporates features common to important energy dissipation problems of various systems. By implementing the sample specific conditions one can extend the model to study various problems. Here we make the following comments: (i) Our work provides an atomic scale theory explaining how and how fast energy dissipates in friction when adsorbates are both small and sparsely distributed. When the adsorbate is small, the adsorbate frequency may lie outside the band of substrate frequencies, and the anharmonic terms that we explicitly take into account here will lead to energy dissipation. Because the adsorbates are distributed sparsely over the substrate, we can neglect the interactions among adsorbates in addressing the energy dissipation from each one. In this case the model can be applied to describe energy dissipation in the boundary lubrication in low coverage of lubricant molecules attached to a surface. For high coverage, one has to consider adsorbate-adsorbate interactions. Our work complements a number of important recent studies that address energy dissipation by phonons through films of lubricants ${ }^{8,9,11}$ and through the collective motion of asperities, ${ }^{6}$ systems where the interactions of the physisorbed or chemisorbed adsorbates give rise to collective excitations involved in friction. (ii) In the model described in Fig. 1, a corrugation in Sample II first increases the potential energy of the adsorbate by squeezing it, but as soon as the corrugation passes over it the adsorbate is suddenly released exciting phonons. The energy of these phonons dissipates until the next corrugation starts to squeeze the adsorbate again. Obviously, this sequence of events gives rise to the stick-slip behavior for the usual elastic constants of the metal samples and adsorbate. We note that the model treated in this work is also suitable for a blunt AFM tip having asperities consisting of many atoms. As for the sharp AFM tip having a single atom at the apex, the model is applied for the apex atom if the local vibration frequency is above the Debye frequency. (iii) Generalizations of the model and the theory presented in this work are relevant to the growth processes of a crystal. After the deposition the adspecies migrate on the surface by dissipating their kinetic energy and eventually they are adsorbed at a local minimum of potential energy. An adatom also moves from one position to the other by the thermal excitation to participate in either epitaxial or island growth. In the course of all these events and processes, the dissipation of the energy of the "hot" atoms is crucial for the atomic scale mechanisms of crystal growth.

In summary, the theory we have presented provides a clear picture of phononic energy dissipation through a nanoparticle, and allows quantitative analysis on this scale. 
${ }^{1}$ For further references see: Physics of Sliding Friction, Vol. 311, Nato ASI Series E, edited by B. N. J. Persson and E. Tosatti (Kluwer, Dordrecht, 1996); Micro/Nanotribology and its Applications, Vol. 330, Nato ASI Series: Applied Sciences, edited by B. Bhushan (Kluwer, Dordrecht, 1997); G. Hähner and N. Spencer, Phys. Today 51, 22 (1998); S. Granick, Phys. Today 52, 26 (1999).

${ }^{2}$ C. M. Mate, G. M. McClelland, R. Erlandson, and S. Chang, Phys. Rev. Lett. 59, 1942 (1987).

${ }^{3}$ B. Bhushan, J. N. Israelachivili, and U. Landman, Nature (London) 347, 607 (1995).

${ }^{4}$ A. P. Sutton and J. B. Pethica, J. Phys.: Condens. Matter 2, 5317 (1990); J. A. Nieminen, A. P. Sutton, and J. B. Pethica, Acta Metall. Mater. 40, 2503 (1992).

${ }^{5}$ M. R. Sorensen, K. W. Jacobsen, and P. Stoltze, Phys. Rev. B 53, 2101 (1996); M. R. Sorensen, K. W. Jacobsen, and H. Jonsson, Phys. Rev. Lett. 77, 5067 (1996).

${ }^{6}$ F. Slanina, Phys. Rev. E 59, 3947 (1999).

${ }^{7}$ V. Zaloj, M. Urbakh, and J. Klafter, Phys. Rev. Lett. 82, 4823 (1999).

${ }^{8}$ M. Cieplak, E. D. Smith, and M. O. Robins, Science 265, 1209 (1994); E. D. Smith, M. O. Robins, and M. Cieplak, Phys. Rev. B 54, 8252 (1996).

${ }^{9}$ J. B. Sokoloff, Phys. Rev. B 42, 760 (1990); Phys. Rev. B 42, 6745(E) (1990); Phys. Rev. B 51, 15573 (1995).

${ }^{10}$ J. B. Sokoloff, Phys. Rev. Lett. 71, 3450 (1993).

${ }^{11}$ M. S. Tomassone, J. B. Sokoloff, A. Widom, and J. Krim, Phys. Rev. Lett. 79, 4798 (1997).

${ }^{12}$ A. Buldum and S. Ciraci, Phys. Rev. B 55, 2606 (1997); 55, 12892 (1997); A. Buldum, S. Ciraci, and I. P. Batra, Phys. Rev. B 57, 2468 (1998); Phys. Rev. B 60, 1982 (1999).
${ }^{13}$ A. Buldum, D. M. Leitner, and S. Ciraci, Phys. Rev. B 59, 16042 (1999).

${ }^{14}$ C. G. Grandqvist, in Springer Proceedings in Physics Vol 62 Surface Science: Lectures on Basic Concepts and Applications, edited by F. A. Ponce and M. Cardona (Springer-Verlag, Berlin, 1991).

${ }^{15}$ R. Schlögl, in Surface Science: Principles and Current Applications, edited by R. J. Mac-Donald, E. C. Taglauer, and K. R. Wandelt (Springer-Verlag, Berlin, 1996).

${ }^{16}$ R. Kaneko, Tribol. Int. 28, 33 (1995).

${ }^{17}$ W. Zhong and D. Tomanek, Phys. Rev. Lett. 64, 3054 (1990); D. Tomanek, W. Zhong, and H. Thomas, Europhys. Lett. 15, 887 (1991).

${ }^{18}$ J. A. Leiro and M. Persson, Surf. Sci. 207, 473 (1989).

${ }^{19}$ B. N. J. Persson, Chem. Phys. Lett. 139, 457 (1987).

${ }^{20}$ B. N. J. Persson and R. Ryberg, Phys. Rev. Lett. 54, 2119 (1985).

${ }^{21}$ F. M. Hoffmann and B. N. J. Persson, Phys. Rev. B 34, 4354 (1986).

${ }^{22}$ B. N. J. Persson and R. Ryberg, Phys. Rev. Lett. 48, 549 (1982).

${ }^{23}$ B. N. J. Persson, J. Phys. C 17, 4741 (1984).

${ }^{24}$ R. Ryberg, Phys. Rev. B 40, 5849 (1989).

${ }^{25}$ A. G. Redfield, Adv. Magn. Reson. 1, 1 (1965); R. K. Wangsness and F. Bloch, Phys. Rev. 89, 728 (1953); F. Bloch, Phys. Rev. 102, 104 (1956).

${ }^{26}$ F. E. Figueirido and M. R. Levy, J. Chem. Phys. 97, 704 (1992); W. T. Pollard and R. A. Friesner, J. Chem. Phys. 100, 5054 (1994); Y.-C. Sun, H. Gai, and G. A. Voth, Chem. Phys. 200, 357 (1995).

${ }^{27}$ Karl Blum, Density Matrix Theory and Applications, 2nd ed. (Plenum Press, N.Y., 1996).

${ }^{28}$ M. R. da Costa, A. O. Caldeira, S. M. Dutra, and H. Westfahl, Jr. (preprint quant-ph/9903022). 